ОЦЕНКА ПСИХОЛОГИЧЕСКИХ ОСОБЕННОСТЕЙ, СОСТАВА ТЕЛА И СТАТУСА ФАКТИЧЕСКОГО ПИТАНИЯ ЖЕНЩИН С НАРУШЕНИЯМИ ПИЩЕВОГО ПОВЕДЕНИЯ

() О.В. Филатова ${ }^{1 *}$, С.С. Половинкин ${ }^{1}$, И.В. Червова², Е.И. Бакланова' , И.О. Плясова

'ФГБОУ ВО «Алтайский государственный университет», г. Барнаул, Россия

ФГБОУ ВО «Алтайский государственный медицинский университет», г. Барнаул, Россия

ОБОСНОВАНИЕ. Нарушения пищевого поведения начали активно изучаться с середины XX в. В последние годы исследователи разных стран находят все новые подтверждения роли пищевого поведения, пищевых привычек пациента в развитии ожирения. Так как сфера исследования пищевого поведения и его нарушений начала активно развиваться сравнительно недавно, многие аспекты этой проблемы еще не изучены.

ЦЕЛЬ. Изучение психологических особенностей, состава тела и параметров фактического питания у женщин с нарушением пищевого поведения.

МЕТОДЫ. Для исследования типов пищевого поведения использовали голландский опросник DEBQ. Для оценки выраженности присущих расстройствам пищевого поведения характеристик использована методика «Шкала оценки пищевого поведения». Актуальное психическое состояние обследуемых изучали с помощью клинико-психологического теста - опросника выраженности психопатологической симптоматики (SCL-90-R). Компонентный состав тела оценивали при помощи аппарата для биоимпедансометрии ABC-01 «Медасс». Оценку фактического питания методом частотного анализа проводили с помощью компьютерной программы «Анализ состояния питания человека».

РЕзУЛьтАТЫ. Во всех группах женщин с нарушениями пищевого поведения выявлены более высокие значения по шкалам стремления к худобе, булимии и неудовлетворенности телом, соматизации, обсессивности-компульсивности, межличностной сензитивности, депрессивности, тревожности. По мере усугубления нарушений пищевого поведения возрастало потребление моно- и дисахаров, добавленного сахара, что сопровождалось увеличением жировой массы тела как в абсолютных, так и в относительных единицах.

ЗАКЛЮЧЕНИЕ. У женщин с нарушениями пищевого поведения выявлены нарушения личностных и психологических характеристик, структуры питания, что сопровождалось увеличением жировой массы тела.

КЛЮЧЕВЫЕ СЛОВА: ожирение, пищевое поведение, биоимпедансометрия, содержание жира в теле, фактическое питание.

\title{
ESTIMATION OF PSYCHOLOGICAL FEATURES, BODY COMPOSITION AND STATUS OF ACTUAL NUTRITION OF WOMEN WITH EATING BEHAVIOR DISORDERS
}

○ Olga V. Filatova ${ }^{1 *}$, Sergei S. Polovinkin¹, Irina V. Chervova², Evgenia I. Baklanova', Irina O. Plyasova

${ }^{1}$ Altai State University, Barnaul, Russia

${ }^{2}$ Altai State Medical University, Barnaul, Russia

BACKGROUND: the violations of eating behavior began to be actively studied since the mid-20th century. In recent years, researchers from different countries have found new evidence of the role of eating behavior, the patient's eating habits in the development of obesity. Since the scope of the study of eating behavior and its disorders began to develop actively relatively recently, many aspects of this problem have not yet been studied.

AIM: was to study the psychological features, body composition and parameters of actual nutrition of women with eating behavior disorders.

METHODS. We used the Dutch questionnaire DEBQ to analyze the types of eating behavior. To assess the severity of eating disorders inherent in eating disorders, the technique "Scale of Eating Behavior Assessment" was used. The actual mental state of the subjects was studied using a clinical and psychological test - a questionnaire of the severity of psychopathological symptoms (SCL-90-R). The component composition of the body was assessed using the apparatus for bioimpedanceometry ABC-01 "Medass". The evaluation of the actual nutrition by the method of frequency analysis was carried out with the help of the computer program "Analysis of the state of human nutrition".

RESULTS. In all groups of women with eating disorders, higher values on the scales of desire for thinness, bulimia and dissatisfaction with the body, somatization, obsessional-compulsiveness, interpersonal sensitivity, depressiveness, anxiety were found. As the eating disorders worsened, the consumption of mono-and disaccharides and added sugar increased, which was accompanied by an increase of the body fat, both in absolute and relative units. 
CONCLUSIONS. In women with eating disorders violations of personal and psychological characteristics, nutrition patterns were detected, which were accompanied by an increase in body fat.

KEYWORDS: obesity, eating behavior, bioimpedanceometry, body fat, actual nutrition.

\section{ОБОСНОВАНИЕ}

Потребность в пище - одна из основных потребностей любого живого существа. Человек, разумеется, не исключение, но, в отличие от многих других животных, у человека на способ приема, вид, состав пищи влияют многочисленные факторы: этнические, географические, религиозные, социальные, медицинские. И как в любом другом виде поведения, в пищевом поведении присутствуют нарушения, которые были выявлены достаточно давно, но начали активно изучаться с середины XX в [1]. В последние годы исследователи разных стран находят все новые подтверждения роли пищевого поведения, пищевых привычек пациента в развитии ожирения [2-5]. Пищевое поведение человека направлено на удовлетворение не только биологических и физиологических, но также его психологических и социальных потребностей [6]. Так как сфера исследования пищевого поведения и его нарушений начала активно развиваться сравнительно недавно [1], многие аспекты этой проблемы еще не изучены.

\section{ЦЕЛЬ}

Изучить психологические особенности, состав тела и параметры фактического питания у женщин с нарушением пищевого поведения.

\section{МЕТОДЫ}

\section{Дизайн исследования}

Проведено поперечное исследование лиц женского пола, проживающих на территории г. Барнаула.

Антропометрические исследования включали измерение длины тела (ДТ), массы тела (МТ), окружности талии (ОТ), окружности бедер (ОБ). Компонентный состав тела оценивали при помощи аппарата для биоимпедансометрии АВС-01 «Медасс», который позволяет определять жировую массу (ЖМТ), тощую безжировую массу (ТМТ), активную клеточную массу (АКМ), массу скелетной мускулатуры (МСМ), общее количество жидкости в организме, внеклеточную жидкость, основной обмен (OO), удельный основной обмен (УОО). Классификацию избыточной массы тела и ожирения проводили по содержанию жира в теле: пониженное, нормальное, повышенное, ожирение для каждого конкретного обследованного в соответствии с его полом и возрастом (например, для женщины 47 лет: пониженное содержание жира - от 20\% до $25 \%$, нормальное - от $25 \%$ до $30 \%$, повышенное - от 30\% до 35\%, ожирение - более 35\%).

Оценку фактического питания методом частотного анализа проводили с помощью компьютерной программы «Анализ состояния питания человека», версия 1.2.4 (ГУ НИИ питания РАМН, 2003-2006 гг.).

При исследовании нарушений пищевого поведения использовали голландский опросник DEBQ [7] для выявления ограничительного, эмоциогенного или экстернального пищевого поведения. Нормативные данные, согласно опроснику DEBQ, по экстернальному пищевому поведению - 2,7, по эмоциогенному пищевому поведению - 1,8, по ограничительному пищевому поведению 2,4. Для оценки выраженности присущих расстройствам пищевого поведения характеристик была использована методика «Шкала оценки пищевого поведения» в адаптации О.А. Ильчик [8]. Актуальное психическое состояние обследуемых изучали с помощью клинико-психологического теста - опросника выраженности психопатологической симптоматики (Simptom Check List-90-Revised - SCL-90-R) [9], ответы на 90 утверждений которого сформировали 9 основных шкал (соматизация, обсессивность-компульсивность, межличностная сензитивность, депрессивность, тревожность, враждебность, фобическая тревожность, паранойяльность, психотизм).

Критерии соответствия

Критериями включения в группу были женский пол, возраст от 36 до 55 лет.

\section{Этическая экспертиза}

Все участники исследования подписывали информированное согласие.

Протокол исследования рассмотрен этическим комитетом, постановили: одобрить представленную статью для направления в журнал «Ожирение и метаболизм». Выписка из протокола № 1 Локального Этического комитета от 30 ноября 2017 года. Заседание состоялось в помещении Алтайского государственного университета по адресу: пр. Ленина, 61.

\section{Статистический анализ}

Принципы расчета размера выборки: размер выборки предварительно не рассчитывался.

Методы статистического анализа данных: статистическая обработка данных проведена с использованием программного продукта SPSS 21.0. Количественные признаки, имеющие нормальное распределение, представлены в виде среднего арифметического (М), ошибки

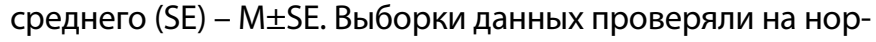
мальность распределения, для чего был использован критерий Колмогорова-Смирнова при уровне значимости $\mathrm{p}<0,05$. Для сравнения двух независимых групп с нормальным распределением использовали однофакторный дисперсионный анализ (ANOVA). Различия значений исследуемых параметров считали статистически значимыми при 95\% пороге вероятности ( $<<0,05)$, на уровне выраженной тенденции при 90\% пороге вероятности $(p<0,1)$, на уровне тенденции при 80\% пороге вероятности $(p<0,2)$. Для выяснения взаимосвязей между изученными показателями проводили корреляционный анализ по Пирсону.

\section{РЕЗУЛЬТАТЫ}

Объекты (участники) исследования

Было обследовано 59 женщин. Обследованные относились ко II периоду зрелого возраста (от 36 до 55 лет) средний возраст 44,6 \pm 0,75 лет. 


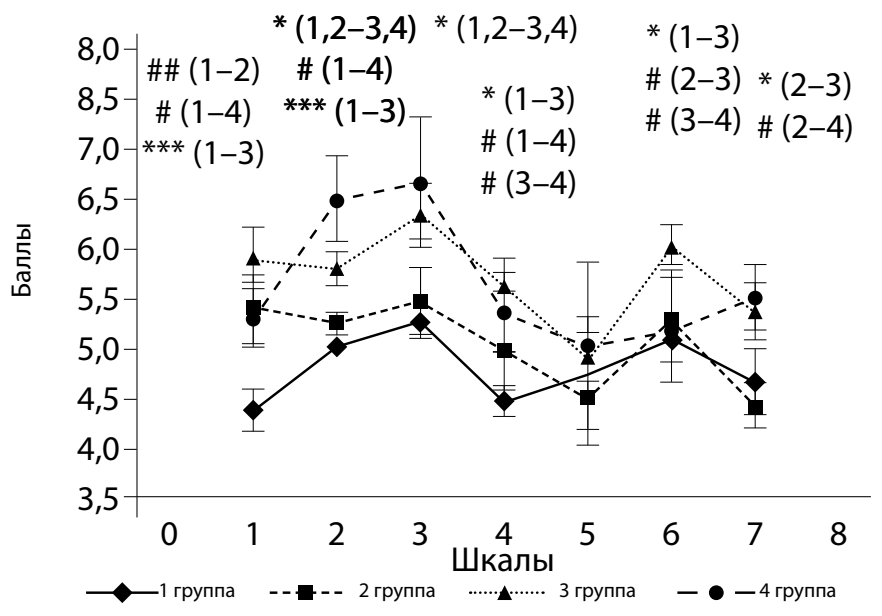

Рис. 1. Показатели опросника «Шкала оценки пищевого поведения» у женщин с нарушениями пищевого поведения.

Примечания: 1 группа - женщины без нарушений пищевого поведения; 2 группа - женщины с нарушением эмоциогенного типа пищевого поведения; 3 группа - женщины с сочетанием нарушения эмоциогенного и ограничительного типов пищевого поведения; 4 группа - женщины с сочетанием нарушения трех типов нарушений пищевого поведения. Шкалы: 1 - стремление к худобе; 2 - булимия; 3 - неудовлетворенность телом; 4 - неэффективность; 5 - перфекционизм; 6 - недоверие; 7 интероцептивная некомпетентность.

\section{Основные результаты исследования}

Обследованные нами женщины были разделены на четыре группы. 20\% (N=12) обследованных не страдали нарушениями пищевого поведения (1-я группа). Нарушения пищевого поведения по результатам голландского опросника выявлены в различных комбинациях у 47 обследованных (80\%). У 22\% (N=13) обследованных женщин выявлено нарушение эмоциогенного типа пищевого поведения (2-я группа). Среди всех испытуемых наиболее часто выявляется сочетание эмоциогенного и ограничительного типов пищевого поведения $(47 \%, \mathrm{~N}=28)$ (3-я группа). У 6 человек (10\%) диагностировано сочетание 3 типов нарушений пищевого поведения (4-я группа).

По результатам опросника «Шкала оценки пищевого поведения» во всех группах женщин с нарушениями пищевого поведения повышено стремление к худобе (рис. 1). В 3-й и 4-й группах выявлены более высокие значения по шкале булимии на статистически значимом уровне. В этих же группах статистически значимо повышен показатель неудовлетворенности телом. Стремление к худобе, в свою очередь, связано положительной связью с показателями неудовлетворенности телом ( $r=0,532$, $p<0,001)$, экстернального $(r=0,363, p=0,005)$ и ограничительного $(r=0,290, p=0,027)$ пищевого поведения.

При сопоставлении степени выраженности психопатологической симптоматики, определяемой с помощью шкалы SCL-90-R, у обследованных выявлены различия по всем шкалам (рис. 2) как минимум на уровне тенденции. В 3-й $(1,0 \pm 0,13)$ и 4-й $(1,1 \pm 0,31)$ группах по сравнению с 1-й $(0,53 \pm 0,12)$ и 2-й $(0,64 \pm 0,16)$ выявлены более высокие значения по шкале соматизации, обсессивно-

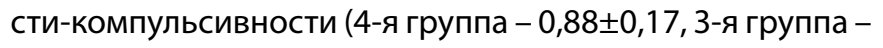

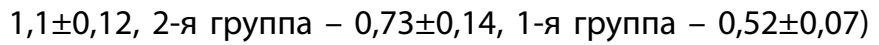
(рис. 2). Средние значения по шкале межличностной сензитивности также оказались выше в 3-й $(0,99 \pm 0,106)$ и 4-й $(1,1 \pm 0,14)$ группах. У обследуемых 3-й $(0,88 \pm 0,11)$ и 4-й $(0,89 \pm 0,22)$ групп определяются более высокие средние баллы по параметру депрессии на уровне тенденции

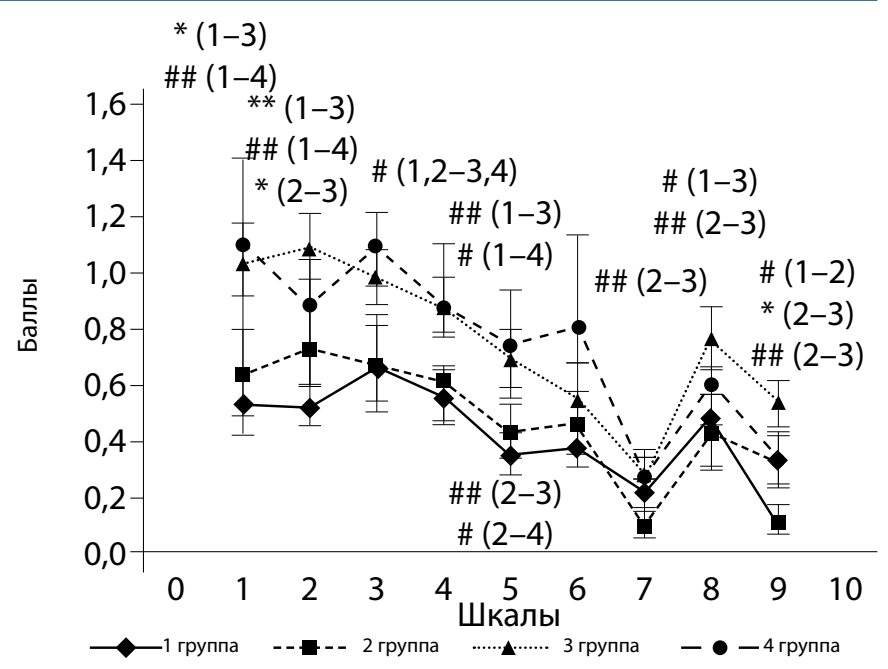

Рис. 2. Показатели SCL-90-R у женщин с нарушениями пищевого поведения.

Примечания: группы - аналогично рис. 1.

Шкалы: 1 - соматизация; 2 - навязчивости; 3 - интерперсональная чувствительность; 4 - депрессия; 5 - тревожность; 6 - враждебность;

7 - фобии; 8 - параноидность; 9 - психотизм.

$\left(\mathrm{p}_{1-3}=0,097, \mathrm{p}_{1-4}=0,200\right)$ по сравнению с 1-й $(0,56 \pm 0,091)$ и 2-й $(0,62 \pm 0,165)$ группами. У обследуемых 3-й и 4-й групп средние значения шкал тревожности и враждебности были выше, чем у представительниц 1-й и 2-й групп (рис. 2).

Общая структура профиля, полученного с помощью опросника выраженности психопатологической симптоматики (SCL-90-R), у женщин с нарушениями пищевого поведения во многом идентична: наиболее выражены показатели по шкалам соматизации, обсессивности-компульсивности, межличностной сензитивности, депрессивности, тревожности. Как наименее выраженные обследуемые всех групп отмечали симптомы, составляющие параметры фобической тревожности, паранойяльности и психотизма (рис. 2).

При исследовании калорийности суточного рациона выявлено максимальное значение энергетической ценности в 4-й группе $(3020,0 \pm 579,57$ ккал) на статистически значимом уровне по сравнению со 2-й группой $\left(1911,0 \pm 232,67\right.$ ккал, $\left.p_{2-4}=0,032\right)$, на уровне выраженной тенденции по сравнению с 3-й группой $\left(2267,1 \pm 161,85\right.$ ккал, $\left.\mathrm{p}_{3-4}=0,111\right)$, на уровне тенденции по сравнению с 1-й группой $(2299,1 \pm 386,06$ ккал, $\left.\mathrm{p}_{1-4}=0,162\right)$. В 4-й группе на уровне тенденции было повышенным потребление белка $(93,1 \pm 20,30$ г) по сравнению с 1-й $(65,5 \pm 10,33$ г, $p=0,138), 2$-й $(62,5 \pm 9,95$ г, $\mathrm{p}=0,098), 3-и ̆ ~(70,7 \pm 7,04$ г, $p=0,186)$ группами. Не было выявлено различий в потреблении жиров. В 4-й группе было повышенным потребление углеводов (337,3 $\pm 75,0$ г) по сравнению с 1-й $(252,8 \pm 24,46$ г, $p=0,174)$, 2-й $(192,5 \pm 27,43$ г, $p=0,020), 3-и ̆ ~(245,6 \pm 26,92$ г, $p=0,107)$ группами. В этой группе на уровне выраженной тенденции максимальным было потребление добавленного сахара $(99,1 \pm 20,98$ г - 4-я группа; 56,7士15,52 г,

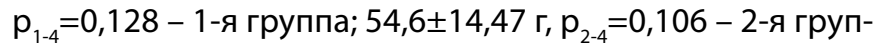
па; 59,4 11,68 г, $p_{3-4}=0,118$ - 3-я группа). От 1-й группы к 4-й возрастало потребление моно- и дисахаров

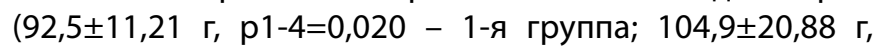
$\mathrm{p}_{2-4}=0,042$ - 2-я группа; $127,6 \pm 16,46$ г, p3-4=0,123 -

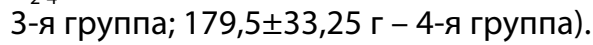

Такой дисбаланс в потреблении простых углеводов нашел свое отражение в увеличении ИМТ от 1-й груп- 


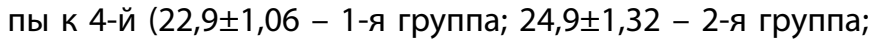
$27,6 \pm 0,98, p_{1-3}=0,006$ - 3-я группа; 34,0 $2,11, p_{1-4}<0,001-$ 4-я группа), что сопровождалось увеличением жировой массы тела как в абсолютных, так и в относительных единицах $(29,4 \pm 1,28 \%$ - 1-я группа; 30,7士2,37\% - 2-я груп-

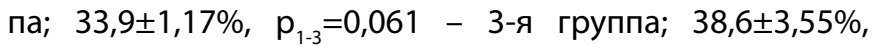
$\mathrm{p}_{1-4}=0,009$ - 4-я группа). Величина нормированного основного обмена на 1 кг ТМТ имеет тенденцию к снижению от 1-й группы к 4-й (32,2士0,67 ккал/кг - 1-я груп-

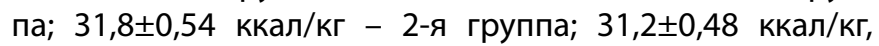
$\mathrm{p}_{1-3}=0,200$ - 3-я группа; 29,5 $\pm 0,55$ ккал/кг, $\mathrm{p}_{1-4}=0,024-$ 4-я группа). Следовательно, причиной повышенной ЖМТ являются особенности пищевого поведения в 3-й и 4-й группах, в 4-й группе - снижение интенсивности ОО и повышенное потребление углеводов.

При проведении корреляционного анализа обнаружено, что с ИМТ, ЖМТ в абсолютных и относительных единицах положительно коррелировало большинство изученных психологических показателей: экстернальное и ограничительное пищевое поведение, стремление кхудобе, булимия, неудовлетворенность телом, недоверие, соматизация, обсессивность-компульсивность, межличностная сензитивность, депрессия, тревожность, враждебность, фобическая тревожность, паранойяльность, психотизм. 35 коэффициентов корреляции соответствовали умеренной $(0,30<r<0,49), 7$ - слабой $(0,20<r<0,29)$, 2 - средней $(0,50<r<0,69)$ силе связи. Максимальная сила связи обнаружена между показателем ЖМТ\% и показателями булимии $(r=0,480, p<0,001)$, неудовлетворенности телом $(r=0,466, p<0,001)$, соматизации $(r=0,510, p<0,001)$, обсессивности-компульсивности $(r=0,449, p<0,001)$, депрессии $(r=0,412, p=0,001)$, тревожности $(r=0,435$, $\mathrm{p}=0,001)$, враждебности $(r=0,428, p=0,001)$, психотизма $(r=0,528, p<0,001)$.

Показаны отрицательные связи между показателем депрессии ( $r=-0,311, p=0,016)$, тревожности ( $r=-0,275$, $\mathrm{p}=0,035)$ и величиной удельного ОО. Величина депрессии также связана отрицательной связью с показателем нормированного ОО $(r=-0,183, p=0,164)$ на уровне тенденции.

Методом корреляционного анализа показаны немногочисленные связи между изученными нами показателями биоимпедансометрии, шкалами опросников и результатами оценки питания. Наибольший интерес представляют положительные корреляционные связи между показателем депрессии и потреблением моно- и дисахаров $(r=0,291, p=0,031)$, крахмала $(r=0,281$, $p=0,038)$, общих углеводов ( $r=0,347, p=0,009)$, а также тревожности и потреблением моно- и дисахаров $(r=0,264$, $\mathrm{p}=0,050)$.

\section{ОБСУЖДЕНИЕ}

\section{Резюме основного результата исследования}

В целом нарушения пищевого поведения выявлены у $80 \%$ обследованных женщин. Наиболее распространенным является сочетание эмоциогенного и ограничительного типов пищевого поведения. Во всех группах женщин с нарушениями пищевого поведения выявлены более высокие значения по шкалам стремления к худобе, булимии и неудовлетворенности телом, соматизации, обсессивности-компульсивности, межлич- ностной сензитивности, депрессивности, тревожности. По мере усугубления нарушений пищевого поведения возрастало потребление моно- и дисахаров, добавленного сахара, что сопровождалось увеличением жировой массы тела как в абсолютных, так и в относительных единицах.

\section{Обсуждение основного результата исследования}

В настоящем исследовании выборка не имеет клинического диагноза нарушений пищевого поведения. Целью было проанализировать, как связаны психогенные факторы с риском возникновения нарушений пищевого поведения у женщин второго периода зрелого возраста.

У обследованных нами женщин с нарушениями пищевого поведения повышено стремление к худобе, неудовлетворенность телом. Вполне возможно, данный феномен носит объективный характер - среднее содержание жира в этих группах соответствует повышенному уровню и ожи-

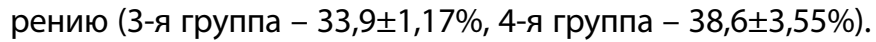
Однако нельзя исключать и влияния социальных факторов. Особое место в формировании неудовлетворенности телом занимают средства массовой информации, через которые происходит интериоризация образов физической привлекательности. Подобные установки часто транслируются на бессознательном уровне, повышая угрозу влияния СМИ на возникновение дисморфофобии и нарушений пищевого поведения [10].

У обследованных женщин с сочетанием нарушений эмоциогенного и ограничительного типов и всех трех типов пищевого поведения обнаруживалась тенденция к депрессии, что подтвердило данные литературы - депрессивные расстройства обнаруживаются у 60-96\% больных пищевыми расстройствами [11], хотя чаще всего речь идет о расстройствах в координате анорексии-булимии.

Оценка фактического питания позволила нам обнаружить дисбаланс в потреблении пищевых веществ в сторону потребления углеводов - в частности, добавленного сахара, моно- и дисахаров. Очевидно, на нарушения пищевого поведения могут влиять не только психогенные, но и биологические факторы. Многочисленные данные свидетельствуют об общих механизмах патогенеза пищевых и аффективных расстройств - в частности, нарушениях нейромедиаторного баланса, в первую очередь, обмена серотонина или 5-гидрокситриптамина. Серотонин играет важную роль не только в развитии нарушений пищевого поведения, но и в регуляции циркадных и сезонных ритмов, механизмах возникновения личностных и сексуальных расстройств, агрессивного поведения. В пользу тесной связи пищевых и аффективных расстройств свидетельствует значительное снижение концентрации в спинномозговой жидкости больных нейропептида 5-НІАА, основного метаболита серотонина, который, по мнению ряда авторов, играет решающую роль в развитии не только пищевых расстройств, но и обсессивно-компульсивных и депрессивных нарушений [11].

\section{Заключение}

Нарушение пищевого поведения у женщин сопровождается изменением психологических признаков, расстройствами приема пищи, что выражалось возрастанием потребления моно- и дисахаров, добавленного 
сахара. Последнее проявлялось увеличением жировой массы тела как в абсолютных, так и в относительных единицах.

\section{Дополнительная информация}

Конфликт интересов. Авторы декларируют отсутствие явных и потенциальных конфликтов интересов, связанных с публикацией настоящей статьи.
Участие авторов. Филатова О.В. - концепция и дизайн исследования, анализ полученных результатов, написание текста; Половинкин С.С. - сбор и обработка материалов, анализ полученных результатов; Червова И.В. концепция и дизайн исследования, редактирование; Бакланова Е.И. - сбор и обработка материалов; Плясова И.О. - сбор и обработка материалов.

Участие авторов. Все авторы внесли значимый вклад в проведение исследования и подготовку статьи, прочли и одобрили финальную версию перед публикацией.

\section{СПИСОК ЛИТЕРАТУРЫ | REFERENCES}

1. Елисеева П.С., Гранская Ю.В. Психологические особенности женщин, переживающих потерю веса // Научные исследования выпускников факультета психологии СпбГу. - 2014. T. 2. - C. 101-108. [Eliseeva PS, Granskaja JuV. Psihologicheskie osobennosti zhenshhin, perezhivajushhih poterju vesa. Nauchnye issledovanija vypusknikov fakul'teta psihologii SpbGU. 2014;2:101108. (in Russ.)]

2. Azagba S, Sharaf MF. Eating Behavior and Obesity in Canada. J. Prim. Care Community Health. 2011;3(1):57-64 doi: $10.1177 / 2150131911417445$.

3. Hays NP, Bathalon GP, McCrory MA, et al. Eating behavior correlates of adult weight gain and obesity in healthy women aged 55-65 y. Am J Clin Nutr. 2002;75(3):476-483. doi: 10.1093/ajcn/75.3.476.

4. Lee HA, Lee WK, Kong K-A, et al. The Effect of Eating Behavior on Being Overweight or Obese During Preadolescence. J Prev Med Public Health. 2011;44(5):226-233. doi: 10.3961/jpmph.2011.44.5.226.

5. Shin A, Lim S-Y, Sung J, et al. Dietary Intake, Eating Habits, and Metabolic Syndrome in Korean Men. J. Am. Diet. Assoc. 2009;109(4):633-640. doi: 10.1016/j.jada.2008.12.015.

6. Соловьева А.В. Факторы риска формирований нарушения пищевого поведения у лиц с избыточной массой тела и ожирением // Медицинский альманах. - 2013. - № 6 - C. 178-180. [Solov'eva AV. Faktory riska formirovanij narushenija pishhevogo povedenija u lic s izbytochnoj massoj tela i ozhireniem. Medicinskij al'manah. 2013;6:178-180. (in Russ.)]

7. van Strien T, Frijters JER, Bergers GPA, Defares PB. The Dutch Eating Behavior Questionnaire (DEBQ) for assessment of restrained, emotional, and external eating behavior. Int. J. Eat. Disord.
1986;5(2):295-315. doi: 10.1002/1098-108x(198602)5:2<295::aideat2260050209>3.0.co;2-t.

8. Ильчик О.А., Сивуха С.В., Скугаревский О.А., Суихи С. Русскоязычная адаптация методики «Шкала оценки пищевого поведения» // Психиатрия, психотерапия и клиническая психология. 2010. - № 3 - C. 4-7. [Il'chik OA, Sivuha SV, Skugarevskij OA, Suihi S. Russkojazychnaja adaptacija metodiki «Shkala ocenki pishhevogo povedenija». Psychiatry, psychotherapy and clinical psychology. 2010;3:4-7. (in Russ.)]

9. Психология посттравматического стресса: практикум / под ред. H.B. Тарабриной. - СПб.: Издательство Питер; 2001. [Tarabrina NV, editor. Psihologija posttravmaticheskogo stressa: praktikum. St. Petersburg: Piter; 2001. (in Russ.)]

10. Дурнева М. Ю., Мешкова Т. А. Влияние социокультурных стандартов привлекательности на формирование отношения к телу и пищевого поведения у девушек подросткового и юношеского возраста // Психологическая наука и образование. - 2013. - № 2 C. 25-34. [Durneva MY, Meshkova TA. Influence of social and cultural standards of attractiveness on the formation of the relationship to the bodyand eating behaviors in adolescent girls and young women. Psychological Science and Education. 2013;(2):25-34. (in Russ.)]

11. Марилов В.В., Артемьева М.С., Сулейманов Р.А., Брюхин А.Е. Результаты длительного лонгитьюдинального исследования нарушений пищевого поведения // Вестник Российского университета дружбы народов. Серия: Медицина. - 2006. - № 2 C. 129-133. [Marilov V, Artemieva MS, Souleimanov RA, Brioukhin AE. Longitudinal study of eating disorders. RUDN Journal of Medicine. 2006;10(2):129-133. (in Russ.)]

\section{ИНФОРМАЦИЯ ОБ АВТОРАХ [AUTHORS INFO]}

*Филатова Ольга Викторовна, д.б.н., профессор [Olga V. Filatova, PhD, Professor] адрес: Россия, 656056, Барнаул, ул. Чернышевского, д. 33 [address: 33 Chernischevskyi street, 656056, Barnaul, Russia]; ORCID: http://orcid.org/0000-0002-4581-5866; eLibrary SPIN: 1979-2220; e-mail: ol-fil@mail.ru

Половинкин Сергей Сергеевич, аспирант [Sergei S. Polovinkin, postgraduate student] ORCID: http://orcid.org/0000-0002-6948-6219; e-mail: sergei.polovinkin@mail.ru

Червова Ирина Васильевна, к.б.н. [lrina V. Chervova, Ph.D.]; ORCID: http://orcid.org/0000-0002-9752-0058; e-mail: irchervova@yandex.ru

Бакланова Евгения Игоревна, студентка [Evgenia I. Baklanova, student]; ORCID: http://orcid.org/0000-0002-3485-2020; e-mail: baklanova.0209@mail.ru

Плясова Ирина Олеговна, студентка [lrina O. Plyasova, student]; ORCID: http://orcid.org/0000-0002-6470-5024; e-mail: i.plyasova@mail.ru

\section{ЦИТИРОВАТЬ:}

Филатова О.В., Половинкин С.С., Червова И.В., Бакланова Е.И., Плясова И.О. Оценка психологических особенностей, состава тела и статуса фактического питания женщин с нарушениями пищевого поведения// Ожирение и метаболизм. - 2018. - Т.15. — №. 3 - С. 28-32. doi: 10.14341/OMET9314

\section{TO CITE THIS ARTICLE:}

Filatova OV, Polovinkin SS, Chervova IV, Baklanova El, Plyasova IO. Estimation of psychological features, body composition and status of actual nutrition of women with eating behavior disorders. Obesity and metabolism. 2018;15(3):28-32.

doi: 10.14341/OMET9314 